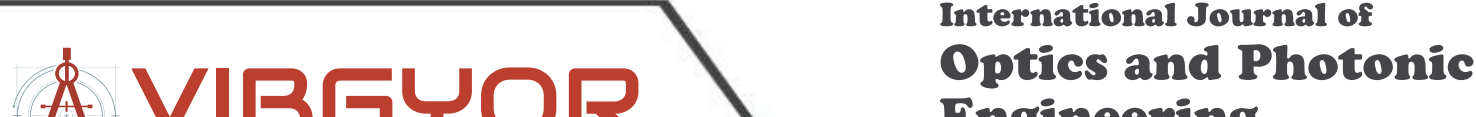 Engineering
}

ISSN: 2631-5092

\section{Enhanced Emission Efficiency of Size-Controlled InGaN/GaN Green Nanopillar Light-Emitting Diodes}

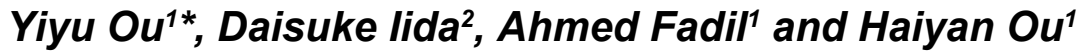 \\ ${ }^{1}$ DTU Fotonik, Technical University of Denmark, Lyngby, Denmark \\ ${ }^{2}$ Department of Applied Physics, Tokyo University of Science, Tokyo, Japan
}

\begin{abstract}
Nanopillar InGaN/GaN green light-emitting diode (LED) arrays were fabricated by self-assembled Au nanoparticles patterning and dry etching process. Structure size and density of the nanopillar arrays have been modified by varying the Au film thickness in the nanopatterning process. Fabricated nanopillar LEDs have been characterized by both room temperature and temperature-dependent photoluminescence measurements. A considerable internal quantum efficiency enhancement was achieved which is attributed to the suppressed quantum confined Stark effect derived from the internal strain relaxation. Meanwhile light extraction efficiency can also be enhanced significantly due to the increased light scattering at nanopillar sidewall. Compared to the planar LED, the nanopillar LED demonstrates the greatest external quantum efficiency enhancement by a factor of 4.08. It is believed that this nanopillar fabrication method can serve as an effective approach to increase the luminescence efficiency of LEDs.
\end{abstract}

\section{Introduction}

In recent years, solid-state lighting devices have attracted a great deal of interest, due to their significant efficiency improvement [1]. Meanwhile, III-nitride compound semiconductors have been widely explored for their applications in light-emitting diodes (LEDs) and great progress has been achieved, leading to commercialization of InGaN/GaN multiple quantum well (MQW) based blue LEDs [2]. However, in order to replace the conventional light sources, LEDs with further improvement in optical performance are expected. One fundamental limit in LEDs is the large spontaneous polarization and piezoelectric fields induced by the internal strain in the MQWs, leading to spatial separation of electrons and holes, known as the quantum confined Stark effect (QCSE), thereby decreases internal quantum efficiency (IQE) and introduces a red-shift in emission wavelength [3-5]. Compared to blue LEDs, green LEDs have much more severe QCSE since the lattice mismatch becomes larger with increased indium content, therefore the internal strain gets larger and then generates the so-called "green gap" phenomenon [6]. Another limit is the low light extraction efficiency (LEE) of LEDs, caused by the large total internal reflection at different material interfaces $[7,8]$. Among different methods, fabrication of nanostructures on LEDs is widely accepted as an effective way not only to relieve the internal strain in MQWs because of their small hetero interfacial areas, but also to enhance the light extraction due to the increased scattering and reduced reflection at the sidewall of the nanostructures $[9,10]$.
Nanostructures can be fabricated or synthesized on GaN based LEDs by various methods, such as bottom-up growth of core/shell nanorod or nanowire structures [10-12], and top-down etched nanopillar structures $[9,13-15]$. The former method usually suffers from higher impurities and point defect densities introduced during growth process under the restricted growth conditions [10,16,17]. In contrast, the nanopillar structures fabricated by the latter way are etched from planar LED thin film structures grown under optimized conditions and can avoid the disadvantages mentioned above. In order to achieve nanopillar LED arrays, a nanopatterning process such as e-beam lithography $[18,19]$, laser holography $[20,21]$, nanoimprint lithography [22], and nanosphere lithography $[9,13,14,23,24]$, is usually required followed by a dry etching process. Compared to these processes, self-assembled metal nanoparticles is a cost-effective, timesaving, and industrially scalable nanopatterning technique $[6,15]$. Nanopillar LED arrays patterned by this method have demonstrated significant efficiency enhancement only in blue LEDs [25-27]. On the other hand, there is an increasing research interest in enhancing the efficiency of green LEDs and very few works has been reported on green LEDs of applying nanopillar structures [28].

In this work, we demonstrated nanopillar LED arrays in InGaN/ GaN green LEDs with tuned pillar size and density, and studied their optical properties. In usual planar LED, external quantum efficiency $(\mathrm{EQE})$ is the product of IQE and LEE, $\mathrm{nEQE}=\mathrm{nIQE} \bullet \mathrm{nLEE}$. For nanopillar LED, the overall luminescence efficiency will also be decided

*Corresponding author: Yiyu Ou, DTU Fotonik, Technical University of Denmark, DK-2800, Lyngby, Denmark, E-mail:yiyo@fotonik.dtu.dk

Received: November 30, 2015: Accepted: January 14, 2016: Published: January 19, 2016

Copyright: ๑ 2016 Ou Y, et al. This is an open-access article distributed under the terms of the Creative Commons Attribution License, which permits unrestricted use, distribution, and reproduction in any medium, provided the original author and source are credited. 
by the active region ratio (ratio of nanopillar LED top surface to total area). So here we rewrite the $\mathrm{EQE}$ as $\mathrm{nEQE}=\mathrm{nIQE} \bullet \mathrm{LEE} \bullet \mathrm{AR}$, where $\mathrm{nAR}$ is the active region ratio to the total area and $\mathrm{nAR}=1$ for planar LED. Metal nanoparticles in the nanopatterning process were formed by rapid thermal processing (RTP) of a thin Au film. By carefully changing the thickness of thin Au film, the average size and density of Au nanoparticles will change accordingly, and later on determines the average size of nanopillar LED structure after dry etching process. The influence of nanopillar LED structure with different size and density on the internal strain relaxation and the IQE of LED was investigated. Au film thickness dependent LEE, EQE, and active region ratio were studied as well. Finally, optimized nanopillar LED structure and its contribution to different optical property were identified.

\section{Sample Fabrication}

Figure 1 shows a schematic illustration of the nanopillar LED fabrication process. The planar InGaN/GaN green LED epiwafer was grown on a c-axis sapphire (0001) substrate by metal-organic chemical vapor deposition. The LED structure has a $3 \mu \mathrm{m}$ Si-doped $\mathrm{n}-\mathrm{GaN}$ layer, followed by five pairs of $\mathrm{InGaN}(2 \mathrm{~nm}) / \mathrm{GaN}(15 \mathrm{~nm})$ MQWs. Then a $130 \mathrm{~nm} \mathrm{Mg-doped} \mathrm{p}-\mathrm{GaN}$ cladding layer is grown on the top. To fabricate nanopillar LED structure, a $150 \mathrm{~nm} \mathrm{SiO}{ }_{2}$ layer was first deposited as an interlayer on the LED sample by plasmaenhanced chemical vapor deposition. Subsequently, a thin Au film (6-11 nm) was deposited on the $\mathrm{SiO}_{2}$ surface by e-beam evaporation. After that the sample was subjected to RTP at $650^{\circ} \mathrm{C}$ for 3 minutes

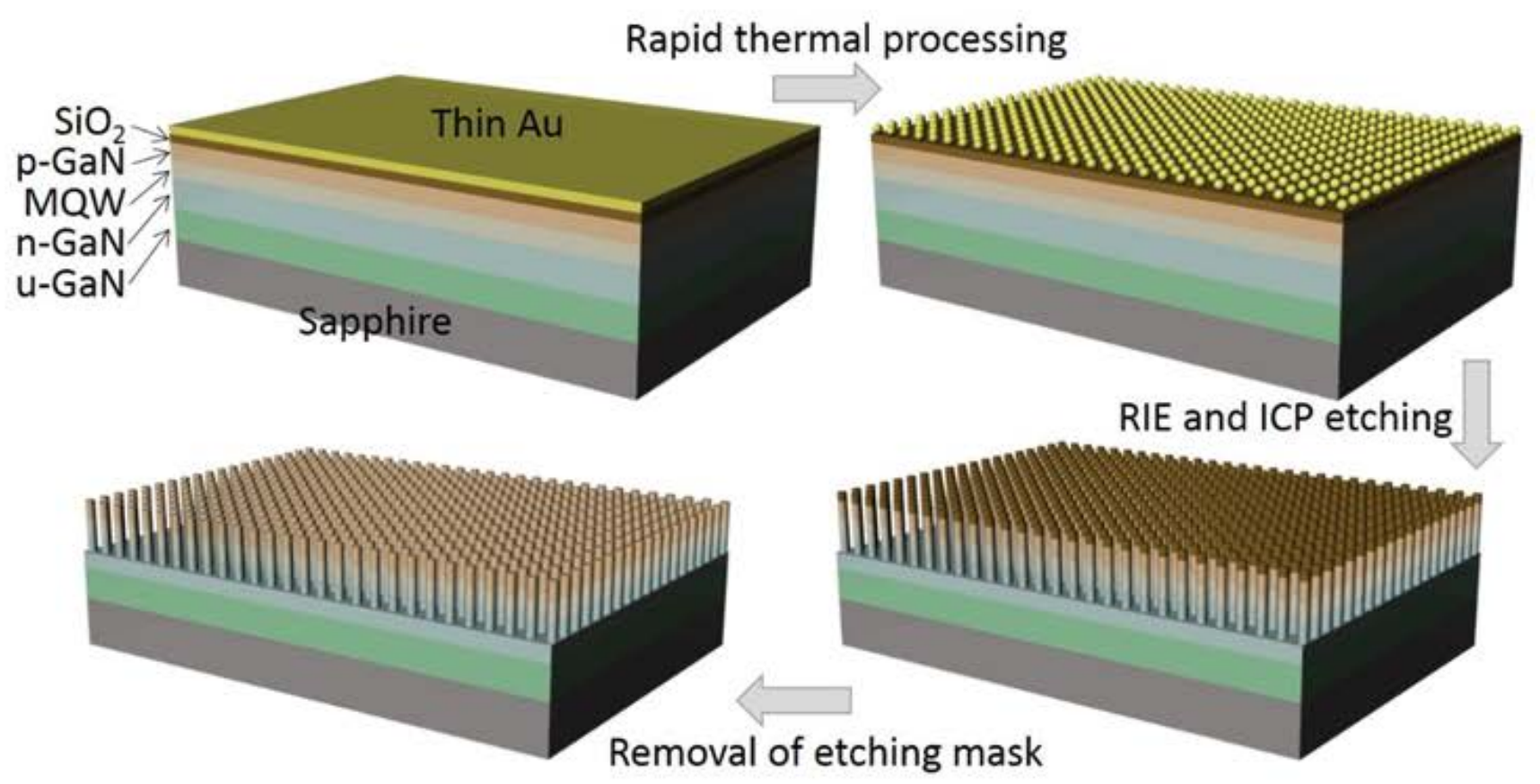

Figure 1: Schematic illustration of the nanopillar LED fabrication process.
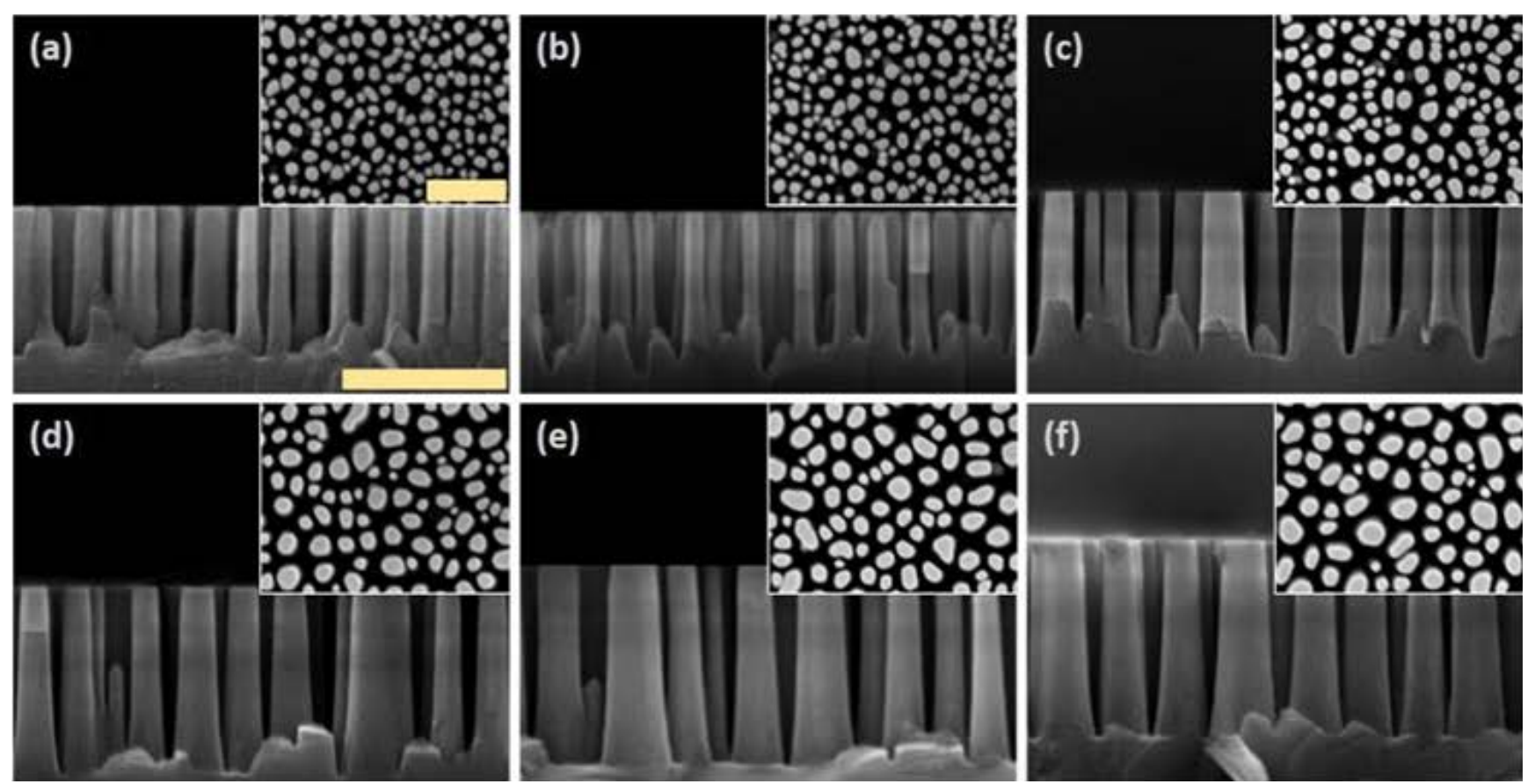

Figure 2 (a-f): Cross-sectional and top-view (inset) SEM images of InGaN/GaN nanopillar structures for LED samples a-f respectively. Both scale bars represent $500 \mathrm{~nm}$.

Citation: Ou Y, lida D, Fadil A, Ou H (2016) Enhanced Emission Efficiency of Size-Controlled InGaN/GaN Green Nanopillar Light-Emitting Diodes. Int J Opt Photonic Eng 1:001 
under $\mathrm{N}_{2}$ ambient to form the self-assembled Au nanoparticles. Reactive-ion etching (RIE) was then conducted to etch the $\mathrm{SiO}_{2}$ using $\mathrm{Au}$ nanoparticles as etch mask. The etching gas was a mixture of $\mathrm{SF}_{6} / \mathrm{O}_{2}$, and residual Au nanoparticles were removed afterwards by Iodine based solution. The LED structure was further etched down to $\mathrm{n}$-GaN layer by inductively coupled plasma (ICP) etching with a gas mixture of $\mathrm{Cl}_{2} / \mathrm{Ar} / \mathrm{BCl}_{3}$, transferring the nanopattern from $\mathrm{SiO}_{2}$ to LED structure. Finally, the residual $\mathrm{SiO}_{2}$ was removed by buffer oxide etchant and nanopillar LEDs were treated in $\mathrm{HCl}$ solution to cure the ICP-induced etching damages on nanopillar sidewall.

A batch of six nanopillar LED arrays: sample a-f, were fabricated by patterns from different Au film thickness: $6 \mathrm{~nm}-11 \mathrm{~nm}$ in a step of $1 \mathrm{~nm}$ applied in the RTP process, respectively. All the samples were etched under the same ICP conditions and etching time. Figure 2 demonstrate the cross-sectional and top-view (inset) scanning electron microscope (SEM) images of nanopillar LED structures for sample a-f respectively. It is seen that, the height of nanopillar LED structure increases with increased $\mathrm{Au}$ film thickness. A nanopillar height of around 460,480, 505, 580, 600, and $600 \mathrm{~nm}$ was achieved from sample a to $\mathrm{f}$ respectively. Although the nanopillar height is slightly different among samples, the truncated nanopillar structures indicate that all the LED structures were not over etched and their active region (MQWs) were fully exposed to the air. So we assume that the contribution difference to optical properties among different nanopillar LEDs was mainly caused by the variation of active region ratio to the total surface which we will discuss later on.

\section{Characterization and Results}

A detailed study of nanopillar structure dependence on Au film thickness was conducted and the cross-sectional size distributions of the nanopillars were presented in figure 3. Here we define the size of each nanopillar as the diameter of a circular structure which has the same area as this nanopillar, and size distribution of different sample were calculated based on this assumption. The LED nanopillar size variation follows a Gaussian distribution for all the samples and the average size of nanopillars increases generally with thicker Au film as we can see from the top-view SEM images in figure 2. Table 1 gives the statistics of nanopillar LED structures in terms of Au film thickness, active region ratio, and nanopillar density, where 'active region ratio' is the ratio of emission area to total area of sample and 'nanopillar density' is the number of nanopillar structures per unit area. One can see that the active region ratios are reduced by more than $51 \%$ for all the nanopillar LEDs while the highest ratio is about $40.7 \%$ of sample d $(9 \mathrm{~nm} \mathrm{Au})$. Such large active region ratio reduction corresponds to a smaller fraction of light emitting region which leads to a decreased emission intensity. It is also noted that the nanopillar density decreases with increased Au film thickness.

Room temperature photoluminescence (RT-PL) spectra were measured using a micro-PL system with a $375 \mathrm{~nm}$ diode laser and demonstrated in figure 4 , where an as-grown planar LED was also measured as a reference sample. To ensure the same optical excitation condition, all the samples were excited from backside (polished sapphire substrate) and detected from top surface. As shown in figure 4, the PL intensity varies among samples. Compared to the reference LED, sample a (6 $\mathrm{nm} \mathrm{Au})$ shows a decreased emission intensity which might attribute to a large surface area. However, when the $\mathrm{Au}$ film thickness increases, the PL intensity of nanopillar LED increases and the largest enhancement with a factor of 4.08 was

Table 1: Statistics of nanopillar LED patterned from rapid thermal processing of thin Au film in terms of Au film thickness, active region ratio, and nanopillar density.

\begin{tabular}{|l|l|l|l|l|l|l|l|}
\hline Sample & ref & a & b & c & d & e & f \\
\hline Au film thickness $(\mathrm{nm})$ & 0 & 6 & 7 & 8 & 9 & 10 & 11 \\
\hline Active region ratio $(\%)$ & 100 & 38.1 & 32.1 & 33 & 40.7 & 37.6 & 37.6 \\
\hline Nanopillar density $\left(1 / \mathrm{\mu m}^{2}\right)$ & n/a & 107 & 101 & 75.2 & 49 & 45.3 & 39.5 \\
\hline
\end{tabular}
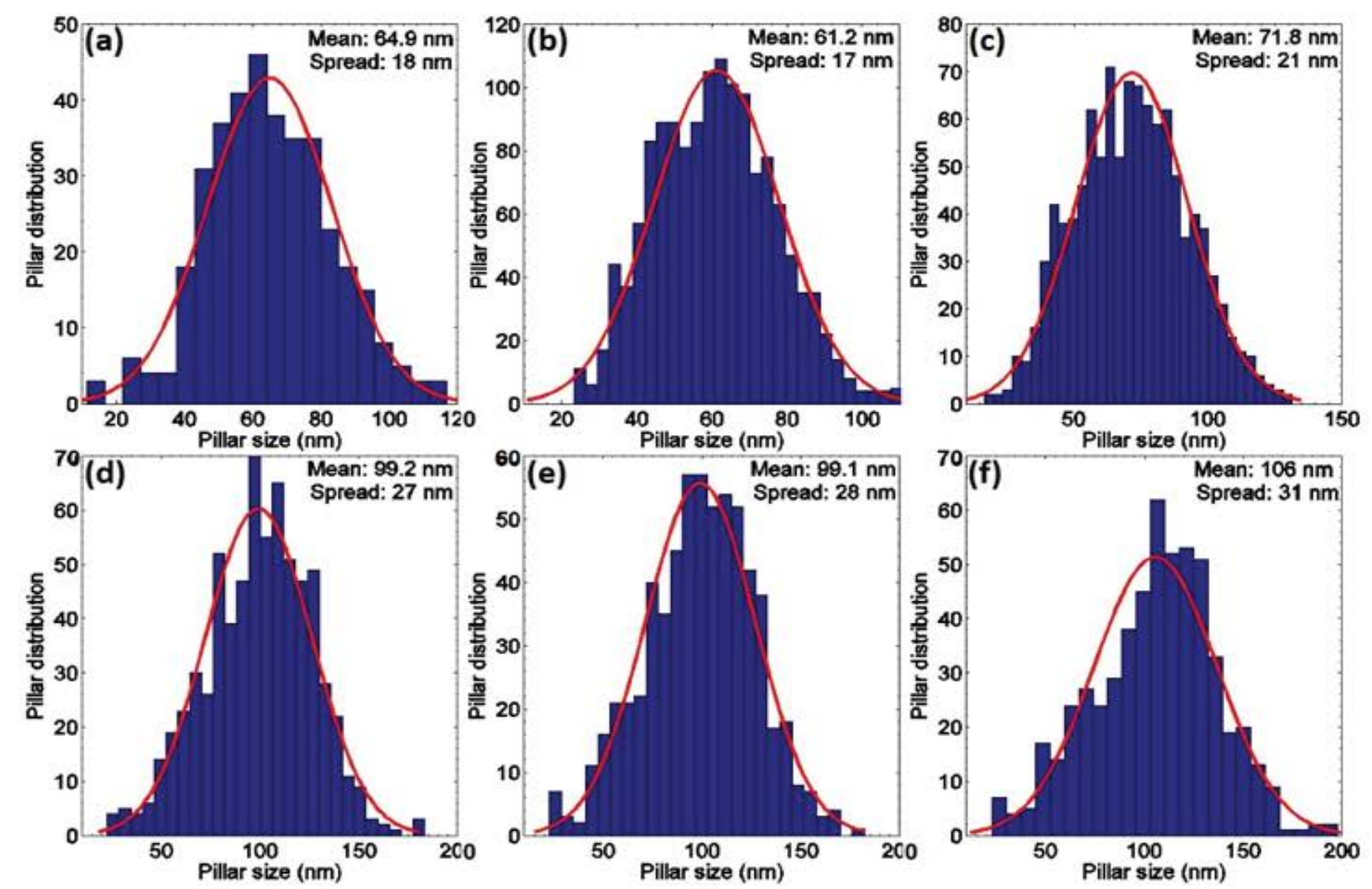

Figure 3 (a-f): Size distribution of the fabricated nanopillars for LED samples a-f respectively. 


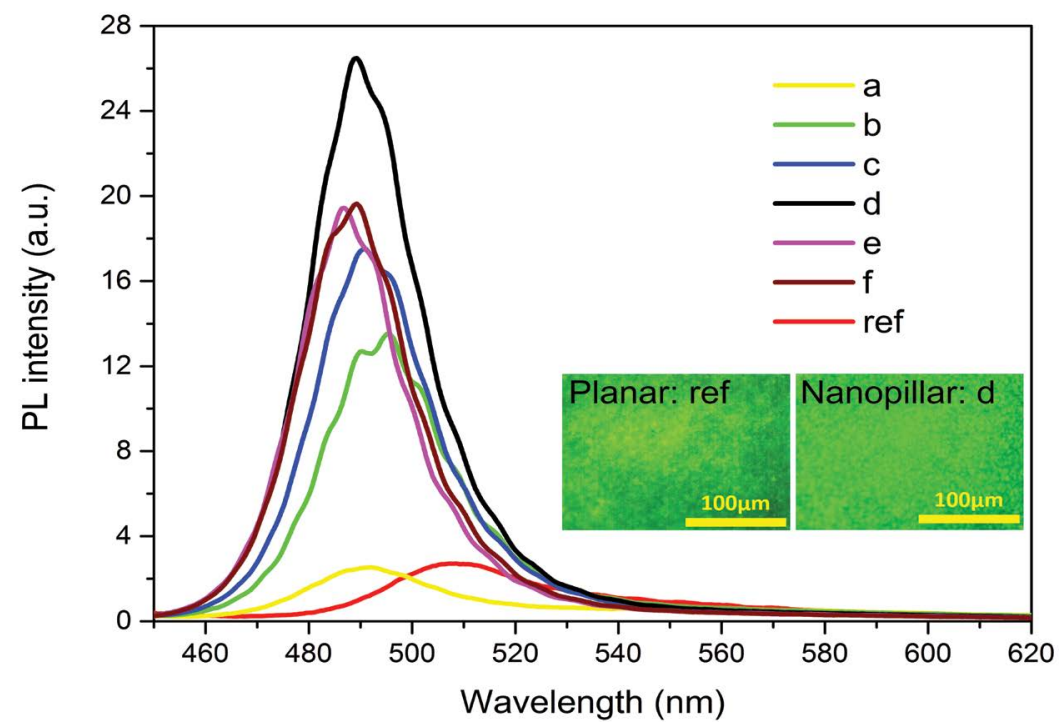

Figure 4: PL intensity spectra of reference and nanopillar LEDs measured at room temperature (inset: optical microscope images of PL emission for the reference LED and sample d).
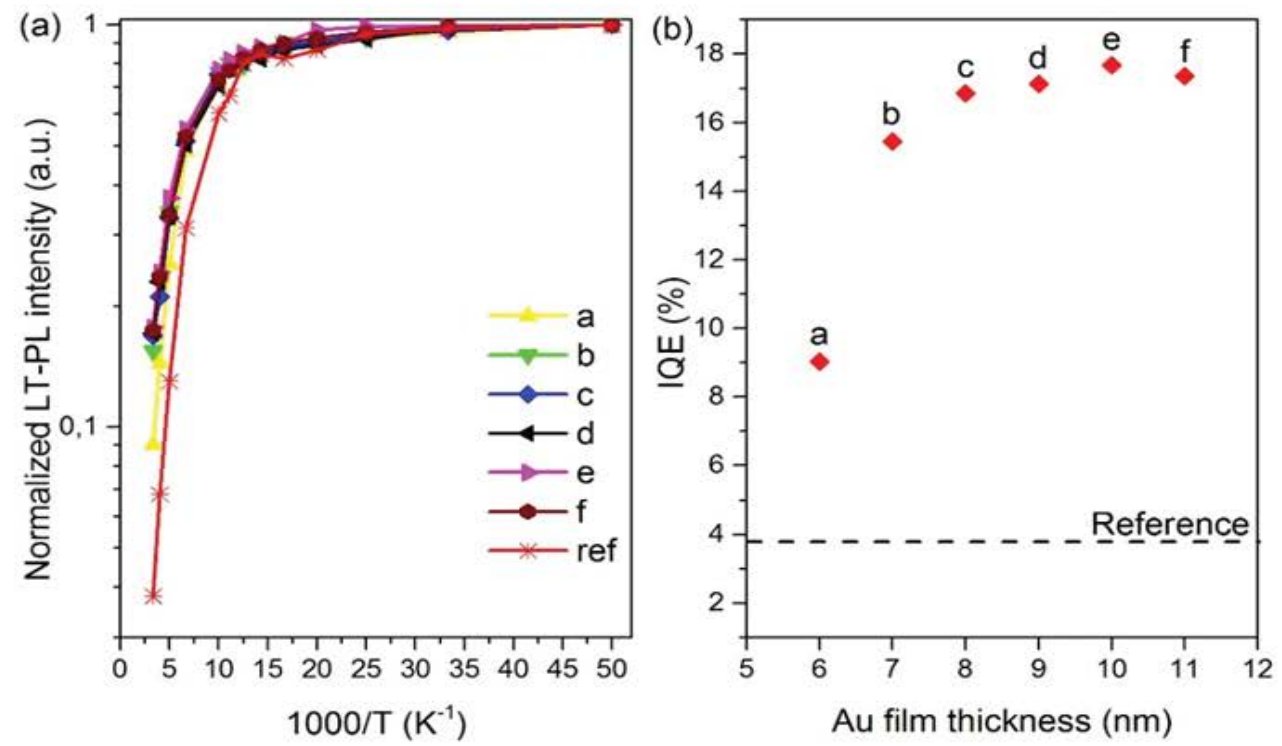

Figure 5: (a) Arrhenius plots of PL intensities measured from 20K to 300K; (b) IQE as a function of Au film thickness for nanopillar LEDs.

achieved at sample d (9 $\mathrm{nm} \mathrm{Au})$. The large PL enhancement was also reflected by the optical microscope images of PL emission in figure 4. Moreover, despite of the PL intensity, all the nanopillar LEDs present a similar blue shift ( 11-14 nm) in the emission wavelength which suggest a reduced QCSE and a large strain relaxation due to the nanopillar fabrication. The reduced QCSE will increase the wave function overlap of electrons and holes and result in an increased IQE. In addition, comparing to similar work in blue LEDs [26,27], we present a larger strain relaxation (blue shift at emission peak) in green nanopillar LEDs, so a larger IQE enhancement could be expected.

To assess the IQE enhancement, temperature-dependent PL measurements were carried out on all the samples by using a cryogenic system (cold heads with closed-cycle helium compressors) and the temperature was varied from $20 \mathrm{~K}$ to $300 \mathrm{~K}$ (room temperature). During all the measurements, an as-grown planar LED was measured under the same conditions as a reference sample. The ratio of the integrated PL intensity at RT to the value at $20 \mathrm{~K}$ is defined as the IQE at RT for each LED sample, assuming that non-radiative recombination is inactivated and IQE is $100 \%$ at $20 \mathrm{~K}$. Figure 5(a) shows the normalized low temperature (LT)-PL intensities as a function of temperature from $20 \mathrm{~K}$ to $300 \mathrm{~K}$ in an Arrhenius plot. It is found that $\mathrm{PL}$ intensities start to decrease when the temperature increases due to the activated non-radiative recombination. Compared to the planar LED, the nanopillar LEDs have non-radiative recombination being activated at a higher temperature which indicates a larger IQE value. The IQE of the nanopillar LEDs and the planar LED were calculated and shown in figure 5(b) as a function of $\mathrm{Au}$ film thickness where all the nanopillar LEDs demonstrate an IQE enhancement. Although sample has a decreased PL intensity at RT, the IQE increased from $3.80 \%$ to $9.03 \%$. When the Au film thickness increased, IQE started to maintain in the same level while the highest IQE of $17.67 \%$ was obtained at sample e (10 $\mathrm{nm} \mathrm{Au})$ which is 4.65 times of the planar LED. The significant IQE enhancement for all the nanopillar LEDs can be attributed to the alleviated QCSE in MQWs and therefore confirm the large strain relaxation that we observed from blue shift at emission peak in RT-PL measurements.

Apart from the active region ratio and IQE, EQE of nanopillar LED is also decided by the LEE. For conventional planar LED, the LEE at top surface can be estimated by $1 / 4 n^{2}$ where $n$ is the refractive index of $\mathrm{GaN}$, thus the LEE of planar LED surface is around 4.0\% [24]. Therefore, 

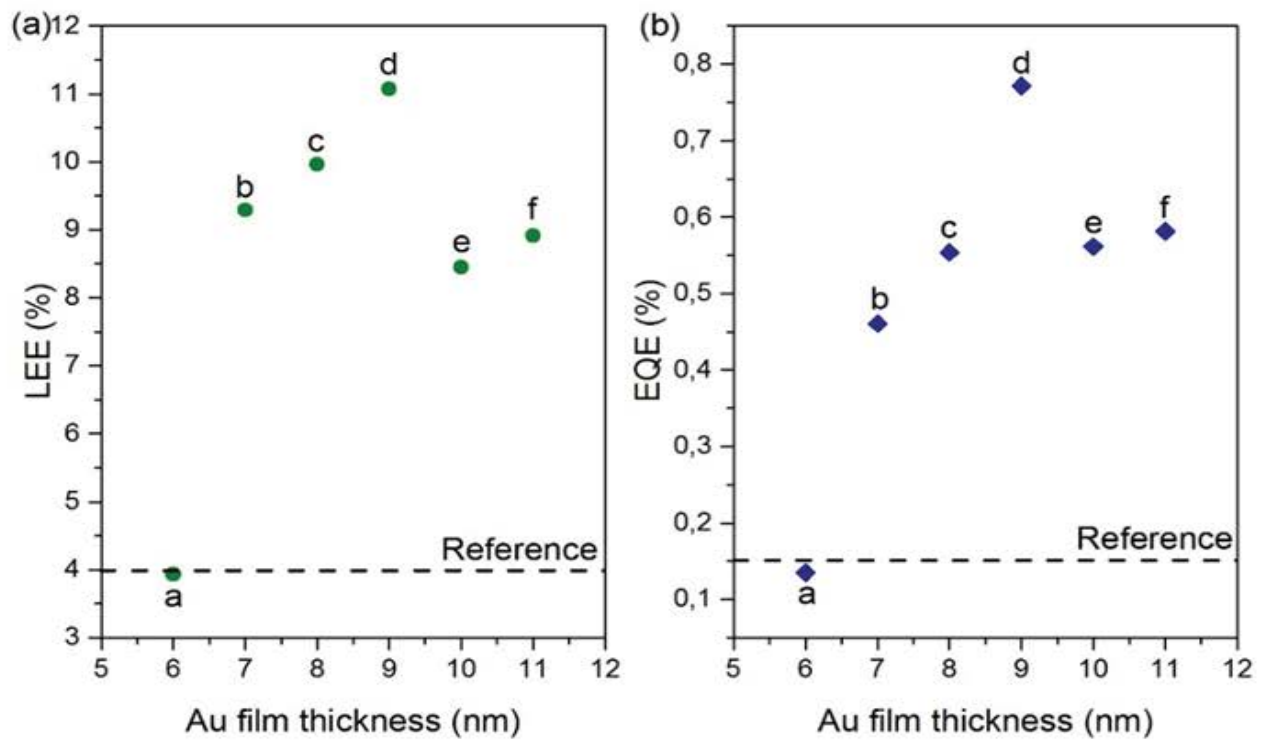

Figure 6: (a) LEE and (b) EQE as a function of Au film thickness for nanopillar LEDs.

Table 2: Summary of IQE, LEE, EQE, and EQE enhancement values of reference and nanopillar LEDs (a-f).

\begin{tabular}{|l|l|l|l|l|l|l|l|}
\hline Sample & ref & a & b & c & d & e & f \\
\hline IQE $(\%)$ & 3.80 & 9.03 & 15.45 & 16.86 & 17.13 & 17.67 & 17.35 \\
\hline LEE (\%) & 4 & 3.93 & 9.29 & 9.96 & 11.07 & 8.45 & 8.91 \\
\hline EQE (\%) & 0.15 & 0.14 & 0.46 & 0.55 & 0.77 & 0.56 & 0.58 \\
\hline EQE enhancement & 0 & -0.11 & 2.04 & 2.65 & 4.08 & 2.70 & 2.83 \\
\hline
\end{tabular}

the EQE of planar green LED can be determined as around $0.15 \%$. The measured RT-PL spectra were recorded by using an optical objective with a large numerical aperture and a short working distance, so here we assume that the detected PL intensities is close to the total surface emission for each LED sample. By knowing the EQE of planar LED and the surface emission intensities of all the LEDs under the same excitation conditions, we can then deduct the EQE of all the nanopillar LEDs. We also know the IQE (from Figure 5(b)) and active region ratio (from Table 1) of all the samples, so the LEE of nanopillar LEDs can also be calculated afterwards. Figure 6 shows the LEE and EQE of nanopillar LEDs as a function of $\mathrm{Au}$ film thickness. One can see that sample a has a similar LEE as the reference planar LED and other nanopillar samples present an obvious LEE enhancement while the largest LEE of $11.07 \%$ was from sample $d$. The same trend has also been found in the EQE plot. The IQE, LEE, EQE and EQE enhancement of reference and nanopillar LEDs have been summarized in table 2. It is noted that an EQE can be enhanced by more than 4 times than the planar LED by fabricating nanopillar LED with a fine structure design.

\section{Conclusion}

In summary, a low-cost, easy processing, top-down approach to fabricate nanopillar LED was adopted in this work to improve the luminescence efficiency of green LEDs. Size controlled nanopillar LED arrays were demonstrated by dry etching with an etch mask pattern from self-assembled $\mathrm{Au}$ nanoparticles. When the Au film thickness increased, Au nanoparticles with lower structure density and larger average size were formed after RTP. Compared to the planar LED, nanopillars LEDs have a much smaller active region ratio which will decrease the luminescence intensity. In addition, a blue shift at emission peak was observed from all the nanopillar LEDs in the PL measurements. This phenomenon was attributed to the suppressed QCSE which is derived from a large built-in strain relaxation. The suppressed QCSE also leads to an increased IQE, and the temperature-dependent PL measurements confirmed the IQE enhancement. EQE and LEE were then calculated for all the samples based on the measured results. Therefore each contribution from the respective IQE, LEE and active region ratio to $\mathrm{EQE}$ was distinguished for each LED sample, hence the optimized nanopillar LED was identified: sample d patterned from $9 \mathrm{~nm}$ Au film with an average pillar size of $99.2 \mathrm{~nm}$, a pillar height of $580 \mathrm{~nm}$, a pillar density of 49 / $\mu \mathrm{m}^{2}$, and an active region ratio of $40.7 \%$. The IQE was increased from $3.80 \%$ to $17.13 \%$ and LEE was increased from $4 \%$ to $11.07 \%$. Finally, a total luminescence intensity enhancement by a factor of 4.08 was achieved with EQE increased from $0.15 \%$ to $0.77 \%$. The promising results suggest that nanopillar LED array is a very effective method to enhance the emission efficiency of green LEDs.

\section{Acknowledgement}

This research was supported by the Danish Council for Strategic Research (0603-00494B). Takahisa Kato from Meijo University is acknowledged for the help on the LT-PL measurement. Y.O. thanks Ms. Yiqi Mao for the assistance on the graphic illustration.

\section{References}

1. Schubert EF, Kim JK (2005) Solid-state light sources getting smart. Science 308: 1274-1278.

2. Siddha Pimputkar, James S. Speck, Steven P. DenBaars \& Shuji Nakamura (2009) Prospects for LED lighting. Nat. Photonics 3: 180-182.

3. Miller DAB, Chemla DS, Damen TC, Gossard AC, Wiegmann W, et al. (1984) Band-Edge Electroabsorption in Quantum Well Structures: The QuantumConfined Stark Effect. Phys. Rev. Lett 53: 2173.

4. Shigefusa F. Chichibu, Akira Uedono1 Takeyoshi Onuma, Benjamin A. Haskell2, Arpan Chakraborty, et al. (2006) Origin of defect-insensitive emission probability in In-containing (AI,In, Ga)N alloy semiconductors. Nat Mater 5: 810-816.

5. TWang, J Bai, S Sakai, JK Ho (2001) Investigation of the emission mechanism in InGaN/GaN-based light-emitting diodes. Appl. Phys. Lett 78: 2617.

6. J Bai, Q Wang, T Wang (2012) Characterization of InGaN-based nanorod light emitting diodes with different indium compositions. J. Appl. Phys 111: 113103.

7. Elison Matioli, Stuart Brinkley, Kathryn M Kelchner, Shuji Nakamura, Steven DenBaars, et al. (2011) Polarized light extraction in m-plane GaN lightemitting diodes by embedded photonic-crystals. Appl. Phys. Lett 98: 251112.

8. Li X.-H, Renbo Song, Ee Y.-K, Kumnorkaew P, Gilchrist J.F, et al (2011) Light Extraction Efficiency and Radiation Patterns of III-Nitride Light-Emitting Diodes With Colloidal Microlens Arrays With Various Aspect Ratios. IEEE Photon. J 3: 489-499.

9. Chong Geng, Tongbo Wei, Xiaoqing Wang, Dezhong Shen, Zhibiao Hao, et al. (2014) Enhancement of Light Output Power from LEDs Based on Monolayer Colloidal Crystal. Small 10, 1668-1686. 
10. Shunfeng Lia, Andreas Waag, (2012) GaN based nanorods for solid state lighting. J. Appl. Phys 111: 071101

11. Fang Qian, Silvija Gradečak, Yat Li, Cheng-Yen Wen, Charles M. Lieber (2005) Core/Multishell Nanowire Heterostructures as Multicolor, HighEfficiency Light-Emitting Diodes. Nano Lett 5: 2287-2291.

12. Riley JR, Padalkar S, Li Q, Lu P, Koleske DD, et al. (2013) Three-dimensional mapping of quantum wells in a GaN/InGaN core-shell nanowire light-emitting diode array. Nano Lett 13: 4317-4325.

13. Qiming Li, Karl R. Westlake, Mary H. Crawford, Stephen R. Lee, Daniel D. Koleske, et al. (2011) Optical performance of top-down fabricated InGaN/ GaN nanorod light emitting diode arrays. Opt. Express 19: 25528-25534

14. Jung Min Lee, Jae Woong Choung, Jaeseok Yi, Dong Hyun Lee, Monica Samal, et al. (2010) Vertical Pillar-Superlattice Array and Graphene Hybrid Light Emitting Diodes. Nano Lett 10: 2783-2788.

15. Zhu Jihong, Wang Liangji, Zhang Shuming, Wang Hui, Zhao Degang, et al (2010) The fabrication of GaN-based nanopillar light-emitting diodes. J. Appl. Phys 108: 074302-074302-4.

16. George T Wang, A Alec Talin, Donald J Werder, J Randall Creighton, Elaine Lai, et al (2006) Highly aligned, template-free growth and characterization of vertical GaN nanowires on sapphire by metal-organic chemical vapou deposition. Nanotechnology 17: 5773.

17. A Alec Talin, George T Wang, Elaine Lai, Richard J Anderson (2008) Correlation of growth temperature, photoluminescence, and resistivity in GaN nanowires. Appl. Phys. Lett 92: 093105.

18. S Keller, C Schaake, NA Fichtenbaum, CJ Neufeld, Y Wu, et al (2006) Optical and structural properties of GaN nanopillar and nanostripe arrays with embedded InGaN GaN multi-quantum wells. J. Appl. Phys 100: 054314

19. V. Ramesh, A. Kikuchi, K. Kishino, M. Funato, Y. Kawakami (2010) Strain relaxation effect by nanotexturing InGaN/GaN multiple quantum well. J. Appl. Phys 107: 114303-114306.
20. Dong-Ho Kim, Chi-O Cho, Yeong-Geun Roh, Heonsu Jeon, Yoon Soo Park, et al. (2005) Appl. Phys. Lett 87: 203508

21. Si-Young Bae, Duk-Jo Kong, Jun-Yeob Lee, Dong-Ju Seo, Dong-Seon Lee (2013) Size-controlled InGaN/GaN nanorod array fabrication and optical characterization. Opt. Express 21: 16854-16862.

22. Hiroshi Ono, Yoshinobu Ono, Kenji Kasahara, Jun Mizuno, Shuichi Shoji (2008) Fabrication of high-intensity light-emitting diodes using nanostructures by ultraviolet nanoimprint lithography and electrodeposition. Jpn. J. Appl. Phys 47: 933-935.

23. Ying-Yuan Huang, Liang-Yi Chen, Chun-Hsiang Chang, Yu-Hsuan Sun, Yun-Wei Cheng, et al. (2011) Investigation of low-temperature electroluminescence of InGaN/GaN based nanorod light emitting arrays. Nanotechnology 22: 045202.

24. Dong P, Yan J, Zhang Y, Wang J, Geng C, et al. (2014) Optical properties of nanopillar AIGaN/GaN MQWs for ultraviolet light-emitting diodes. Opt Express 22: A320-327.

25. Dae-Woo Jeon, Won Mook Choi, Hyeon-Jin Shin, Seon-Mi Yoon, Jae-Young Choi, et al. (2011) Nanopillar InGaN/GaN light emitting diodes integrated with homogeneous multilayer graphene electrodes. J. Mater. Chem 21: 1768817692.

26. C H Chiu, T C Lu, H W Huang, C F Lai, C C Kao, et al. (2007) Fabrication of InGaN/GaN nanorod light-emitting diodes with self-assembled Ni metal islands. Nanotechnology 18, 445201.

27. Zhu Ji-Hong, Zhang Shu-Ming, Sun Xian, Zhao De-Gang, Zhu Jian-Jun, et al. (2008) Fabrication and Optical Characterization of GaN-Based Nanopillar Light Emitting Diodes. Chin. Phys. Lett. 25, 3485.

28. Zhuang Z, Guo X, Liu B, Hu F, Dai J, et al. (2016) Great enhancement in the excitonic recombination and light extraction of highly ordered InGaN/GaN elliptic nanorod arrays on a wafer scale. Nanotechnology 27: 015301. 